\title{
Theoretical Studies on the Electronic Properties and the Chemical Bonding of Transition Metal Complexes using DFT and Ligand Field Theory
}

\author{
Mihail Atanasov ${ }^{\star}$ and Claude Daul
}

\begin{abstract}
The research activity within our laboratory of computational chemistry at the University of Fribourg is presented. In this review, a brief outline of a recently proposed Ligand Field Density Functional Theory (LFDFT) model for single nuclear and its extension to dimer transition metal complexes is given. Applications of the model to dinuclear complexes are illustrated for the interpretation of exchange coupling in the bis- $\mu$-hydroxo-bridged dimer of $\mathrm{Cu}(I)$ and to the description of the quadruple metal-metal bond in $\mathrm{Re}_{2} \mathrm{Cl}_{8}{ }^{2-}$. The analysis of the chemical bonding is compared with results obtained using other approaches, i.e. the Extended Transition State model and the Electron Localization Function. It is shown that the DFT supported Ligand Field Theory provides consistent description of the ground and excited state properties of transition metal complexes.
\end{abstract}

Keywords: Density Functional Theory · Electronic absorption and emission spectra · Ligand Field Theory . Magnetic-exchange coupling $\cdot$ Transition metal dimer complexes

\section{Introduction}

An intrinsic feature of transition metal ions (TMI) is the rather localized character of their $3 \mathrm{~d}$ electrons and this property, for example of free ions like $\mathrm{Cr}^{3+}, \mathrm{Cu}^{2+}$, is preserved in their complexes, although modified by covalency however. This allows the metal-ligand interaction to be formulated as being mainly ionic and the metal-ligand bond to be interpreted as a donor-acceptor

${ }^{*}$ Correspondence: Prof. Dr. M. Atanasov

Département de Chimie

Departement für Chemie

Ch. du Musée 9

$\mathrm{CH}-1700$ Fribourg

Tel.: +41263008743

Fax: +41263009738

E-Mail: mihail.atanasov@unifr.ch bond. In simple terms, the ligand donates electrons into the empty valence shell of the TMI - the partly filled $3 \mathrm{~d}$ and the empty $4 \mathrm{~s}$ and $4 p$ shells, which leads to metal-centered antibonding and ligand-centered bonding molecular orbitals (MOs), but these interactions are assumed to be weak enough to be treated by perturbation theory. Then, electronic transitions giving rise to absorption and emission spectra in the visible region are located within the many-electron states which originate from a well defined $\mathrm{d}^{\mathrm{n}}$-configuration of the TMI. Ligand-tometal charge transfer (LMCT) transitions are not included in this manifold and need a different treatment (see below). All these features, which are mostly born out by interpretation of experiment, define what we call a 'Werner' type complex. This group of compounds is mainly restricted to TMI in their normal oxidation states and to ligand anions like $\mathrm{F}, \mathrm{Cl}, \mathrm{Br}$ or molecules such as $\mathrm{H}_{2} \mathrm{O}, \mathrm{NH}_{3}$. Recently, a new model to treat their electronic structure has been proposed in our group - the Ligand-Field Density Functional Theory (LFDFT). Its mathematical formalism has been described in great detail elsewhere [1-3]. Before going on to discuss in simple terms what LFDFT is, let us first ask why we should use DFT for TMI complexes and can we restrict the calculations of their ground and excited state electronic structure to a DFT treatment only?

\section{A Motivation for DFT}

In 1964 Hohenberg and Kohn [4] stated that there exists a universal functional of the electron density, which is independent of the chemical system (accounted for by an external potential) and for which the ground state energy has its correct minimal value for the system. In the formulation of their theorem, the electron density was considered spin free. One year later, Kohn and Sham provided a route to a set of working equations [5], similar to those of Hartree and Hartree-Fock. These Kohn-Sham (KS) equations allow, for systems with slowly varying density (i.e. not far from a homogeneous electron gas) the calculation of the electron density self-consistently, starting from a reasonable guess. The theory allows, finally, the calculation of the electronic energy and other electronic properties for the system from the known electron density.

In writing down these one-electron equations, Kohn and Sham started from a reference system of non-interacting electrons and introduced exchange and Coulomb correlation using an approximate effective potential - the exchange correlation potential. It also considers the non-additivity of the kinetic energy of the electrons due to their interactions. Subsequently, the Kohn-Sham equations became the building block of all user-oriented modern, molecu- 
lar orbital-based DFT programs (KSDFT). In fact, approximate functionals of improving quality have been proposed and applied with considerable success to thermochemistry, to predict: molecular geometry, force fields, infra red (IR), nuclear magnetic resonance (NMR), photoelectron, electron spin resonance (ESR), ultraviolet (UV) spectra and the reactivity of closed shell organic and inorganic (including TMI) systems [6-7].

A great merit of the DFT is its ability to account for electronic correlation, which is important for TMI complexes, at comparably low computational costs. This allows the calculation of systems of significantly larger size than wave function based methods. However, for open-shell systems, the spin-density exhibits, in general, a lower symmetry than the electronic Hamiltonian (Kohn-Sham or Hartree-Fock); this is because of the spin-polarization yielding spin-densities which are no longer invariant with respect to the rotations of the symmetry point group. For example in the case of atoms, $\alpha$ (or $\beta$ ) spin possesses cylindrical symmetry and violates the spherical symmetry e.g. in cases of the $\mathrm{C}$ atom $\left({ }^{3} \mathrm{P}\right.$ ground state).

Even larger problems are encountered in TMI in complexes of high symmetry (cubic, octahedral, tetrahedral). Here, orbitally degenerate ground states, for example of $\mathrm{CuF}_{6}{ }^{4-}\left({ }^{2} \mathrm{E}\right)$ or $\mathrm{MnF}_{6}{ }^{3-}\left({ }^{5} \mathrm{E}-\right.$ high-spin $)$, or excited states $-\mathrm{CrCl}_{6}{ }^{3-}\left({ }^{4} \mathrm{~T}_{2}\right)-$ all with an octahedral geometry, cannot be described by DFT. Instead, a constraint DFT procedure is applied: an average occupation of each degenerate orbital set $\left(e, t_{2}\right)$ is used in order to provide proper symmetry of the space part of the electron density, and a spin-restricted open shell procedure is adopted using non-integer and equal number of $\alpha$ and $\beta$ spins in order to ensure correct spin-symmetry of the system.

However, at variance to the spin-restricted open-shell Hartree-Fock theory, Roothaan's spin-coupling operator [8], which is state and symmetry dependent, is replaced by an orbital independent exchange-correlation potential e.g. DFT codes, such as ADF [9]. Current- and spindensity functional theory for inhomogeneous electronic systems in strong magnetic fields have been developed a long time ago [10]. Unfortunately practical implementations are lacking when compared to the theoretical developments. The symmetry problem seems to be solved in the openshell localized Hartree-Fock approach to the exact-exchange Kohn-Sham treatment of open-shell atoms and molecules proposed by Sala and Görling [11]. However, Coulomb correlation, which, for TMI dominates the exchange correlation and the necessity to account for non-dynamic correlation (via Configuration Interaction
(CI), see below) still remains beyond the reach of the present DFT methodology. We can conclude that DFT alone is not able to account for both the electronic structure of the ground and the excited states of transition metal complexes and one has to resort to other methods or to a combination of different methods.

\section{Electronic Structure Models for TMI Complexes: Ligand Field Theory}

Ligand field theory is an approximate theory, essentially applicable to mononuclear metal complexes. In this theory, the valence electrons are separated into two sets: (i) active electrons occupying d- (or f-) orbitals and (ii) passive electrons occupying ligand orbitals. The following approximations are made:

a) The interactions of electrons in set (i) are treated exactly as in free ions;

b) The energies of electrons in set (ii) are sufficiently low compared to the energies of the d-(or f-) orbitals that their effect is merely to shield the nuclear charges;

c) The effect of the passive electrons in set (ii) on the active d-(or f-) electrons in set (i) is represented by an effective potential (or pseudopotential) called the ligand field potential which is generally represented in the basis of the $\mathrm{d}$ - (or $\mathrm{f}$-) orbitals as $\left\langle\chi_{i}|h| \chi_{j}\right\rangle$, where $\chi_{\mathrm{i}}, \chi_{\mathrm{j}}$ are either d- or f-orbitals (or both).

Thus in ligand field theory [12-13], one focuses on the $\mathrm{d}^{\mathrm{n}}$-configuration and considers the effective Hamiltonian Eqn. (1). It includes the one electron effective ligand field Hamiltonian h(i) (consisting of kinetic and potential energy for each electron) and the

$H=\Sigma_{(i)} h(i)+\Sigma_{(i, j)} G(i, j)$

two-electron G(i,j) operator which takes account of the Coulombic interactions between d-electrons (via the $1 / \mathrm{r}_{\mathrm{ij}}$ operator); summation is carried out over the delectrons $\mathrm{i}>\mathrm{j}$. We note that the operators $\mathrm{h}(\mathrm{i})$ and $\mathrm{G}(\mathrm{i}, \mathrm{j})$ are effective and various LF models differ in the way they approximate these operators. We expand the total wavefunction in a basis of Slater determinants (SD) - 45, 120, 210, and 252 SD for $\mathrm{n}=2(8), 3(7), 4(6)$ and 5 . When acting on the $\mathrm{SD}$, the operator $\mathrm{H}$ leads to oneand two-electron matrix elements, $\mathrm{h}_{\mathrm{ab}}$ and $\mathrm{G}_{\mathrm{abcd}}$ (Eqn.(2)). In Eqn. (1) and Eqn. (2) the symbols $\mathrm{i}, \mathrm{j}(1,2)$ label electrons and the symbols a,b,c and d label orbitals, respectively. In the central field approximation the $\mathrm{G}_{\mathrm{abcd}}$ integrals are:

$$
\begin{aligned}
& h_{a b}=\int a^{*}(1) h(1) b(1) d \tau_{1} \\
& \mathrm{G}_{\mathrm{abcd}}=\iint \mathrm{a}^{*}(1) \mathrm{b} *(2) \mathrm{G}(1,2) \mathrm{c}(1) \\
& d(2) d \tau_{1} d \tau_{2}
\end{aligned}
$$

expressed in terms of the Racah parameters $\mathrm{A}, \mathrm{B}$, and $\mathrm{C}$, pertaining to the spherical symmetry but reduced from the free ion values by covalency (nephelauxetic effect). The ligand field does account for the symmetry lowering from spherical to the molecular point group of the complex due to the chemical environment. Thus, in ligand field theory the influence of the ligands on the TMI is described totally by the $5 \times 5$ ligand field matrix $h_{a b}$ whose matrix elements reflect both electrostatic(crystal field) and covalent(overlap) perturbations from the coordinated ligands and thus the chemical features of the complex. For an octahedral complex and a basis of real dorbitals, for example, the $5 \times 5$ matrix is a diagonal with matrix elements of $\varepsilon_{\mathrm{e}}=(3 / 5)$ 10Dq for the $\mathrm{e}_{\mathrm{g}}\left(\varepsilon=\mathrm{d}_{\mathrm{x} 2-\mathrm{y} 2}, \theta=\mathrm{d}_{\mathrm{z} 2}\right)$ and $\varepsilon_{\mathrm{t}}=-(2 / 5) 10 \mathrm{Dq}$ for the $\mathrm{t}_{2 \mathrm{~g}}\left(\xi=\mathrm{d}_{\mathrm{yz}}, \eta=\mathrm{d}_{\mathrm{zx}}\right.$ and $\zeta=d_{x y}$ ) orbitals obeying the baricenter rule $\left(2 \varepsilon_{\mathrm{e}}+3 \varepsilon_{\mathrm{t}}=0\right)$. The $\varepsilon_{\mathrm{e}}-\varepsilon_{\mathrm{t}}$ orbital energy difference is nothing but the cubic ligand-field splitting parameter 10Dq, which is a positive quantity, because of the stronger/weaker $\sigma / \pi$ interactions of the $e_{g} / t_{2 g}$ orbitals. The d-orbital splitting is smaller and opposite in a tetrahedral complex, where the metal-ligand interactions for $\mathrm{e}$ and $\mathrm{t}_{2}$ orbitals are of $\pi$ and $\sigma+\pi$ type, respectively. Thus, for a $\mathrm{d}^{2}$ complex the total of the 45 electronic states is fully described in terms of three parameters only, $10 \mathrm{Dq}, \mathrm{B}$, and C. The many-electron diagram (Tanabe-Sugano diagram, Fig. 1) has been applied with success to interpret electronic spectra of $\mathrm{d}^{2}$-oxo-anions such as $\mathrm{CrO}_{4}{ }^{4-}, \mathrm{MnO}_{4}{ }^{3-}$, $\mathrm{FeO}_{4}{ }^{2-}$ [14-16], which also allows the determination of the parameters $10 \mathrm{Dq}, \mathrm{B}$, and $\mathrm{C}$ from the spectra.

\section{The LFDFT and its Extension to Di-Nuclear TMI Complexes}

The LFDFT model [1-2] is based on a multi-determinant description of the multiplet structures originating from the $\mathrm{d}^{\mathrm{n}}$ configuration of the TMI in the surrounding of coordinating ligands by combining the $\mathrm{CI}$ and the KS-DFT approaches. In doing so, both dynamical correlation (via the DFT exchange-correlation potential) and nondynamical correlation (via CI) is considered. The latter one accounts for the rather localized character of the d-electron wavefunction. The key feature of this approach is the explicit treatment of near degeneracy effects (long-range correlation) using ad hoc configuration interaction (CI) within the active space of Kohn-Sham (KS) orbitals 


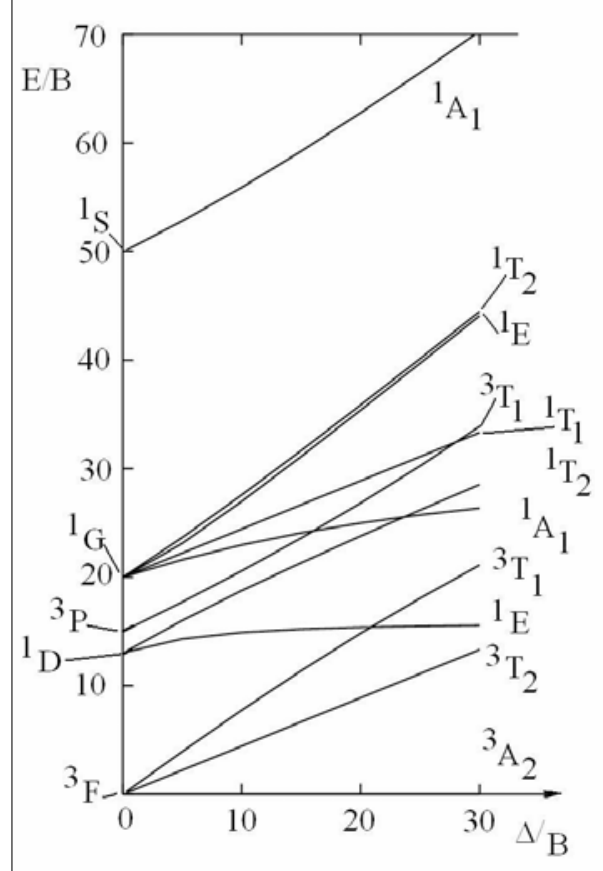

Fig. 1. Tanabe-Sugano diagram for a tetrahedral $\mathrm{d}^{2}$ complex $(\mathrm{C} / \mathrm{B}=4.0)$

with dominant d-character. The calculation of the CI-matrices is based on an analysis of the energies calculated according to KSDFT of the single determinants (microstates) constructed from frozen Kohn-Sham orbitals. The LFDFT procedure consists of the following steps:

(i) A spin-restricted KSDFT- Self Consistent Field (SCF) calculation corresponding to an Average-Of-Configuration $(\mathrm{AOC}) \mathrm{d}^{\mathrm{n}}$, where an $\mathrm{n} / 5$ occupation of each orbital is carried out. This insures that all active electrons (d- or $\mathrm{f}$-) are treated on an equal footing and the interelectronic repulsion is regarded as spherical. In doing so, we gather all the chemical information connected with the ligand field of symmetry lower than spherical into the one electron $(5 \times 5)$ LF matrix.

(ii) Using the Kohn-Sham orbitals from the first step and occupying them adequately, the energies of all SD are calculated.

(iii) Finally, the SD energies are utilized (using a script, written in MATLAB) to obtain all needed model parameters - the $5 \times 5$ ligand field matrix and the parameters B and C - in a leastsquares fit. Comparing SD energies from DFT with those calculated using LF parameter values, we can state for all considered cases that the LF parameterization scheme is remarkably compatible with the SD energies from DFT; standard deviations between the two sets of SD energies (DFT and LFT) are found typically between 0.02 and $0.1 \mathrm{eV}$.
These parameters are then used in a full CI ligand field program to calculate energies and electronic properties of all multiplets split out of a $\mathrm{d}^{\mathrm{n}}$ configuration. Symmetry analysis is supported by the program, however it is a great merit of this approach that it is able to calculate systems of symmetry as low as $\mathrm{C}_{1}$. This makes the approach suitable to bio-inorganic problems including for example active sites in enzymes. In particular, the $5 \times 5$ LF matrix can easily be deduced from a single AOC DFT calculation. We have shown [2] that the matrix of the LF, resulting from the many electron treatment is essentially the same (differences between two set of data not exceeding 1-2\%) as the one obtained using the following simple recipe [17]:

Let us denote KS-orbitals dominated by d-functions which result from an AOC $\mathrm{d}^{\mathrm{n}}$ KSDFT-SCF calculation with column vectors $\vec{V}_{i}$ and their energies by $\varepsilon_{i}^{K S}$, the latter defining the diagonal matrix E. From the components of the eigenvector matrix built up from such columns one takes only the components corresponding to the $d$ functions. Let us denote the square matrix composed of these column vectors by $\mathbf{U}$ and introduce the overlap matrix $\mathbf{S}$ :

$\mathbf{S}=\mathbf{U}^{\mathrm{T}} \mathbf{U}$

Since $\mathbf{U}$ is in general not orthogonal, we use Löwdin's symmetric orthogonalization scheme to obtain an equivalent set of orthogonal eigenvectors $(\mathbf{C})$ :

$$
\mathbf{C}=\mathbf{U S}^{-\frac{1}{2}}
$$

We identify now these vectors with the eigenfunctions of the effective LF Hamiltonian $h_{\mathrm{LF}}^{\text {eff }}$ and we seek:

$$
\varphi_{\mathrm{i}}=\sum_{\mu=1}^{5} \mathrm{c}_{\mu \mathrm{i}} \mathrm{d}_{\mu}
$$

and $\varepsilon_{\mathrm{i}}^{\mathrm{KS}}=\left\langle\varphi_{\mathrm{i}}\left|\mathrm{h}_{\mathrm{LF}}^{\text {eff }}\right| \varphi_{\mathrm{i}}\right\rangle$ with the corresponding eigenvalues. The $5 \times 5$ LF matrix $\mathbf{V}_{\mathbf{L F}}=\left\{\mathrm{h}_{\mu \nu}\right\}$ is given by:

$$
\mathbf{V}_{\mathbf{L F}}=\mathbf{C ~ E ~} \mathbf{C}^{\mathbf{T}}=\left\{\mathrm{h}_{\mu v}\right\}=\left\{\sum_{\mathrm{i}=1}^{5} \mathrm{c}_{\mu \mathrm{i}} \varepsilon_{\mathrm{i}}^{\mathrm{KS}} \mathbf{c}_{\mathrm{vi}}\right\}
$$

Remarkably, the matrix $\mathbf{V}_{\mathbf{L F}}$ is obtained in a general form without any assumptions (such as is done in Crystal Field Theory (CFT) or in the Angular Overlap Model $(\mathrm{AOM}))$ and accounts for both electrostatic and covalent contributions to the ligand field. Moreover, being determined in a variational DFT-SCF procedure, it circumvents assumptions based on perturbation theory. It is particularly suited to cases of low symmetry and complex coordination geometries where application of CFT or AOM, because of the large number of model parameters, is not easy.

The LFDFT approach has been applied and validated using well-documented spectra and structures on octahedral and tetrahedral TM complexes [1-3]. An extension of this method allows the estimation of spin-orbit coupling constants [18] and for the calculation of $g$ - and fine structure A-tensors in EPR [19]. With the estimation of spin-orbit coupling constants, treatment of the zero-field splitting [20] and of the paramagnetic contributions to NMR shielding constants becomes possible [21]

In the following, the extension of the LFDFT method to transition metal dimer complexes [22][23] is briefly reviewed. Let us assume that two semi-occupied orbitals $\mathrm{dl}_{1}$ and $\mathrm{dl}_{2}$ located on both symmetry equivalent fragments couple to yield an in-phase (a) and an out-of-phase (b) MO (Eqn. (7)).

$$
\begin{aligned}
& \mathrm{a}=\frac{1}{\sqrt{2}}\left(\mathrm{dl}_{1}+\mathrm{dl}_{2}\right) \\
& \mathrm{b}=\frac{1}{\sqrt{2}}\left(\mathrm{dl}_{1}-\mathrm{dl}_{2}\right)
\end{aligned}
$$

where $\mathrm{a}$ and $\mathrm{b}$ belong to two different irreps. Moreover we neglect here the overlap between $\mathrm{dl}_{1}$ and $\mathrm{dl}_{2}$. Six micro-states or Single Determinants (SD) result. Two are doubly occupied $l a^{+} a^{-}|,| b^{+} b^{-} \mid$and four are singly occupied $\left|a^{+} b^{-}\right|,\left|a^{+} b^{+}\right|, \mid a^{-} b^{+}$, , $\left|a^{-} b^{-}\right|$. The doubly occupied SD having $\mathrm{a} \otimes \mathrm{a}=\mathrm{b} \otimes \mathrm{b}=$ A spatial symmetry, correspond to closed shells and are spin singlets. The SD based on singly occupied spin orbitals have $a \otimes b=B$ spacial symmetry and correspond to a singlet and to a triplet. The two SD with $M_{S}=0:\left|a^{+} b^{-}\right|$ and $\mathrm{la}^{-} \mathrm{b}^{+}$, belong both to a singlet and to a triplet. The energies of all these determinants can be calculated from DFT. Let us denote their energies by

$$
\begin{aligned}
& \mathrm{E}_{1}=\mathrm{E}\left(\left|\mathrm{a}^{+} \mathrm{a}^{-}\right|\right), \\
& \mathrm{E}_{2}=\mathrm{E}\left(\left|\mathrm{b}^{+} \mathrm{b}^{-}\right|\right), \\
& \mathrm{E}_{3}=\mathrm{E}\left(\left|\mathrm{la}^{+} \mathrm{b}^{+}\right|\right)=\mathrm{E}\left(\left|\mathrm{a}^{-} \mathrm{b}^{-}\right|\right), \\
& \mathrm{E}_{4}=\mathrm{E}\left(\mathrm{la}^{+} \mathrm{b}^{-} \mid\right)=\mathrm{E}\left(\mathrm{la}^{-} \mathrm{b}^{+} \mid\right)
\end{aligned}
$$

We note that the difference $\mathrm{E}_{4}-\mathrm{E}_{3}$ equals the exchange integral [ablab] which is also the quantity accounting for the mixing (1:1 in the limit of a full localization) between the $1 \mathrm{a}^{+} \mathrm{a}^{-} \mid$and $\left|\mathrm{b}^{+} \mathrm{b}^{-}\right|$microstates. This leads to the secular Eqn. (9) which after diagonalization 


$$
\left[\begin{array}{cc}
\mathrm{E}_{1} & \left(\mathrm{E}_{4}-\mathrm{E}_{3}\right) \\
\left(\mathrm{E}_{4}-\mathrm{E}_{3}\right) & \mathrm{E}_{2}
\end{array}\right]
$$

yields the eigenvalues $E_{-}$and $E_{+}$and the energy separation between the lowest singlet state and the triplet $\mathrm{E}_{-}-\mathrm{E}_{3}$, referred to as the singlet-triplet spitting. The latter is identical to the exchange integral in magnetism. It is a good measure for the covalence stabilization of a bonding electron pair with respect to a non-bonding triplet pair. When compared in the limit of complete dissociation these quantities yield the total bonding energy.

Let us now consider the formation of bonding in terms of a localized model for bonding. Within such a model ( $c f$. Anderson [24]), $\mathrm{dl}_{1}$ and $\mathrm{dl}_{2}$ are singly occupied in the ground state for separate fragments giving rise to a triplet and to a singlet with wave functions $\psi_{\mathrm{T}}$ and $\psi_{\mathrm{S}}$ (Eqn. (10) and (11), respectively). There are two further singlet states $\psi_{\mathrm{S}}^{\mathrm{CT}}$ and $\psi_{\mathrm{S}}^{\prime \mathrm{CT}}$ arising when either of the two magnetic electrons is transferred to the other magnetic orbital (SOMO), i.e.

$$
\begin{aligned}
\psi_{T}= & \left|d l_{1}^{+} d l_{2}^{+}\right| ;\left|d l_{1}^{-} d l_{2}^{-}\right| ; \frac{1}{\sqrt{2}}\left(\left|d l_{1}^{+} d l_{2}^{-}\right|+\mid\right. \\
& \left.d l_{1}^{-} d l_{2}^{+} \mid\right),
\end{aligned}
$$

$$
\begin{aligned}
& \psi_{S}=\frac{1}{\sqrt{2}}\left(\left|d l_{1}^{+} d l_{2}^{-}\right|-\left|d l_{1}^{-} d l_{2}^{+}\right|\right), \\
& \psi_{S}^{C T}=\frac{1}{\sqrt{2}}\left(\left|d l_{1}^{+} d l_{1}^{-}\right|+\left|d l_{2}^{+} d l_{2}^{-}\right|\right), \\
& \psi_{S}^{C T}=\frac{1}{\sqrt{2}}\left(\left|d l_{1}^{+} d l_{1}^{-}\right|-\left|d l_{2}^{+} d l_{2}^{-}\right|\right),
\end{aligned}
$$

where $\psi_{\mathrm{S}}$ lies by $2 \mathrm{~K}_{12}$ at higher energy than $\psi_{\mathrm{T}}$. We take the energy of the latter state as reference $\left\{\mathrm{E}\left(\psi_{\mathrm{T}}\right)=0\right\} . \mathrm{K}_{12}$ is the classical Heisenberg exchange integral,

$$
\begin{aligned}
& K_{12}=\iint d l_{1}(1)^{*} d l_{2}(1) \frac{1}{r_{12}} d l_{1}(2) d l_{2}(2)^{*} d \tau_{1} d \tau_{2} \\
& =\left[\mathrm{dl}_{1} \mathrm{dl}_{2} \mid \mathrm{dl}_{1} \mathrm{dl}_{2}\right]
\end{aligned}
$$

which is always positive. It reflects the exchange stabilization of the triplet over the singlet due to gain in potential energy connected with the spatial extension of the Fermi (exchange) hole (potential exchange). The $\psi_{\mathrm{S}}$ two-electron wave-function can mix with the charge transfer state $\psi_{\mathrm{S}}^{\mathrm{CT}}$. Its energy, denoted U, equals the difference between the Coulomb repulsions of two electrons on the same center, i.e. $\left|\mathrm{dl}_{1}^{+} \mathrm{dl}_{1}^{-}\right|$or $\left|\mathrm{dl}_{2}^{+} \mathrm{dl}_{2}^{-}\right| \quad\left(\mathrm{U}_{11}=\left[\mathrm{dl}_{1} \mathrm{dl}_{1}\right.\right.$ $\left.\left.\mid \mathrm{dl}_{1} \mathrm{dl}_{1}\right]=\mathrm{U}_{22}=\left[\mathrm{dl}_{2} \mathrm{dl}_{2} \mid \mathrm{dl}_{2} \mathrm{dl}_{2}\right]\right)$ and when they are located on different centers (the notation $\mathrm{U}_{12}=\left[\mathrm{dl}_{1} \mathrm{dl}_{1} \mid \mathrm{dl}_{2} \mathrm{dl}_{2}\right]$ applies). Thus the energy separation between the $\mathrm{dl}_{1}{ }^{2}$ (or $\mathrm{dl}_{2}^{2}$ ) excited state and the $\mathrm{dl}_{1}{ }_{1}^{1} \mathrm{dl}_{2}{ }^{1}$ ground state configurations is:

$$
\mathrm{U}=\mathrm{U}_{11}-\mathrm{U}_{12}
$$

$\mathrm{U}$ is also a positive quantity. The interaction matrix element between $\psi_{\mathrm{S}}$ and $\psi_{\mathrm{S}}{ }^{\mathrm{CT}}$ (Eqn. (14)) reflects the delocalization of the bonding electrons due to orbital overlap. The quantity $\mathrm{t}_{12}=<\mathrm{dl}_{1}|\mathrm{~h}| \mathrm{dl}_{2}>$ is referred to as the transfer (hopping) integral between the two sites. Thus, we obtain:

$$
\left\langle\psi_{\mathrm{S}}|\mathrm{H}| \psi_{S}^{C T}\right\rangle=2 \mathrm{~T}_{12}=2\left(\mathrm{t}_{12}+\left[\mathrm{dl}_{1} \mathrm{dl}_{1} \mid \mathrm{dl}_{1} \mathrm{dl}_{2}\right]\right)
$$

Calculations show that $\mathrm{T}_{12}=\mathrm{t}_{12}$ in a very good approximation, differences being generally less than $0.002 \mathrm{eV}$. This term tends to lower the singlet- over the triplet-energy and is intrinsically connected with the gain of kinetic energy (kinetic exchange). The interaction matrix (Eqn. (15a)) describes the combined effect of these two opposite interactions. Using perturbation theory one obtains Eqn. (15b) for the singlet-triplet

$$
\begin{aligned}
& \psi_{S} \\
& \psi_{S}^{C T} \\
& {\left[\begin{array}{cc}
2 K_{12} & 2 T_{12} \\
2 T_{12} & U+2 K_{12}
\end{array}\right]} \\
& \left(E_{S}-E_{T}\right)_{P}=J_{12}^{P}=J_{12}^{f}+J_{12}^{a f}=2 K_{12}-\frac{4 T_{12}^{2}}{U}
\end{aligned}
$$

energy separation $J_{12}^{p}$, i.e. the exchange integral. As has been pointed out in [25], the parameters $\mathrm{K}_{12}$, $\mathrm{U}$ and $\mathrm{T}_{12}$ can be expressed in terms of the Coulomb integrals $\left(\mathrm{J}_{\mathrm{aa}}, \mathrm{J}_{\mathrm{bb}}\right.$ and $\left.\mathrm{J}_{\mathrm{ab}}\right)$, exchange integral $\mathrm{K}_{\mathrm{ab}}$ and of $\varepsilon(b)-\varepsilon(a)$, the KS-orbital energy difference. Eqns. (16)-(18) below, resume these relations:

$$
K_{12}=\frac{1}{4}\left(J_{a a}+J_{b b}-2 J_{a b}\right)=\frac{1}{4}\left(E_{1}+E_{2}-2 E_{4}\right)
$$

$$
\mathrm{U}=\mathrm{U}_{11}-\mathrm{U}_{12}=2 \mathrm{~K}_{\mathrm{ab}}=2\left(\mathrm{E}_{4}-\mathrm{E}_{3}\right)
$$

$$
T_{12} \cong \frac{1}{2}\{\varepsilon(a)-\varepsilon(b)\}=\frac{1}{4}\left(E_{2}-E_{1}\right)
$$

We would like to point out that these expressions are furthermore related to the energies of the single determinants $1 \mathrm{a}^{+} \mathrm{a}^{-} \mid$, $\left|b^{+} b^{-}\right|,\left|a^{+} b^{+}\right|,\left|a^{+} b^{-}\right|$(i.e. $\mathrm{E}_{1}, \mathrm{E}_{2}, \mathrm{E}_{3}$ and $\mathrm{E}_{4}$ respectively).

Thus, Eqns. (16)-(18) allow us to obtain $\mathrm{K}_{12}$, $\mathrm{U}$ and $\mathrm{T}_{12}$ directly from DFT. We get therefore a bonding model in terms of localized orbitals, whose parameters are readily obtained from the DFT SD energies $\mathrm{E}_{1}, \mathrm{E}_{2}, \mathrm{E}_{3}$, and $\mathrm{E}_{4}$ of the dinuclear complex. It is remarkable that the same model can be applied with success, both to magnetic exchange coupling and to bond analysis. This makes it possible to consider magnetic and bonding phenomena on the same footing. In fact, there is no fundamental difference between antiferromagnetism and chemical bonding.

\section{Applications}

\subsection{Exchange Splitting in $\mathrm{Cu}(\mathrm{OH})_{2} \mathrm{Cu}$ Dimers}

The usual pattern of an exchange coupling between pairs of TMI with open shells is an anti-ferromagnetic spin-alignment corresponding to a weak delocalization of unpaired spin-density from one center to another center, i.e. that of a weak covalent bond as described by the term: $-4 \mathrm{~T}_{12}{ }^{2} / \mathrm{U}$, Eqn. (15b). It outweighs the contribution of the first term $\left(2 \mathrm{~K}_{12}\right)$, the latter tending to lower exchange (Pauli) repulsion between electrons with parallel spins. It has been therefore challenging to find systems where the latter effect dominates, leading to ferromagnetic spin-alignments. This is the case if magnetic orbitals are orthogonal to each other or nearly so; a situation encountered in edge-sharing square planes or octahedra with $\mathrm{M}_{1}-\mathrm{X}-\mathrm{M}_{2}$ bridging angles $\beta$ close to $90^{\circ}$ [26].

An illustration of this is given by bisbipyridyl- $\mu$-dihydroxo-dicopper (II) nitrate with a $\mathrm{Cu}-\mathrm{OH}-\mathrm{Cu}$ bridging angle of $95.6^{\circ}$ and an exchange coupling constant $\mathrm{J}_{12}=$ $0.021 \mathrm{eV}$ [27]. A DFT-LDA geometry optimization using a $\left[\left(\mathrm{NH}_{3}\right)_{2} \mathrm{CuOH}\right]_{2}{ }^{2+}$ model cluster leads to a geometry of the bridging $\mathrm{Cu}(\mathrm{OH})_{2} \mathrm{Cu}^{2+}$ moiety very close to the experimental finding (Fig. 2). Unpaired electrons on $\mathrm{Cu}^{2+}$ are characterized by a $\mathrm{d}_{\mathrm{x} 2-\mathrm{y} 2}$ ground state which is weakly affected by long axial contacts to $\mathrm{NO}_{3}^{-}$, which we neglect here. The exchange coupling constant $\mathrm{J}_{12}=0.021 \mathrm{eV}$ calculated by LFDFT matches perfectly well the experimental value, but deviates from the prediction of an antiferromagnetic coupling given by the broken symmetry (BS) DFT approach [28] $\left(\mathrm{J}_{12}{ }^{\mathrm{BS}}=-0.099 \mathrm{eV}\right)$.

In Fig. 3, we compare energies of the four independent Slater determinants as given by our procedure with the state energies after taking the $\left|a^{+} a^{-}\right|-\left|b^{+} b^{-}\right|$configu- 
rational mixing into account. The former configuration is stabilized by localization leading to a final singlet state, but it does not cross (in contrast to the usual case) the triplet term T. Experimental data show [27] that $\mathrm{J}_{12}$ becomes strongly antiferromagnetic when the $\mathrm{Cu}-\mathrm{O}-\mathrm{Cu}$ bridging angle $(\beta)$ is increased by structural manipulations allowing one to tune the magnetic properties. Thus the increase of the value of $\beta$ to $104.1^{\circ}$ in $[\mathrm{Cu}(\text { tmen }) \mathrm{OH}]_{2} \mathrm{Br}\left(\right.$ tmen $=\mathrm{N}, \mathrm{N}, \mathrm{N}^{\prime}, \mathrm{N}^{\prime}-$ tetramethylethylenediamine) agrees with the reported negative value of $\mathrm{J}_{12}(-0.063 \mathrm{eV})$ [26]. Antiferromagnetism for this geometry is also obtained by LFDFT, but the resulting value exceeds now the experimental value by a factor of 2.88 (however the BSDFT value is off by a factor of 4.61). The reason is that DFT leads systematically to lower values for the energy $U$, which causes an increase of the $-4 \mathrm{~T}_{12}{ }^{2} / \mathrm{U}$, in cases where this

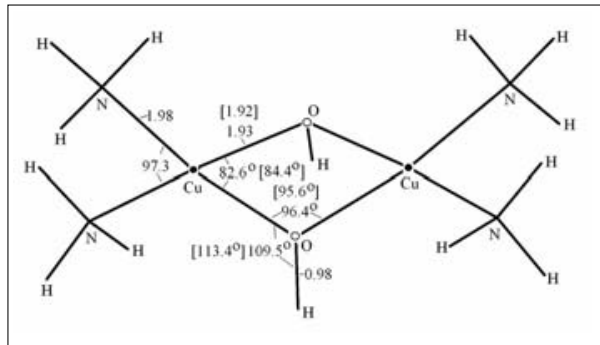

Fig. 2. Bond distances (in $\AA$ ) and bond angles (in $\left.{ }^{\circ}\right)$ from a DFT geometry optimization (spinunrestricted, $S=M_{S}=1$, LDA-VWN functional, non-relativistic TZP basis, $\mathrm{Cu}-2 \mathrm{p}, \mathrm{O}-1 \mathrm{~s}, \mathrm{~N} 1-$ $\mathrm{s}$, frozen cores) of a $\left[\mathrm{Cu}\left(\mathrm{NH}_{3}\right)_{2}(\mathrm{OH})\right]_{2}{ }^{2+}$ model cluster and experimental parameters (in square brackets) as reported from X-ray diffraction study of bis-bipyridil- $\mu$-dihydroxo-dicopper(II) nitrate $\left[\mathrm{Cu}\left(\mathrm{C}_{10} \mathrm{H}_{8} \mathrm{~N}_{2}\right)(\mathrm{OH})-\left(\mathrm{NO}_{3}\right)_{2}\right]$, R.J. Majeste, E.A. Meyers, J. Phys.Chem. 1970, 74, 3497. term plays an important role (see [22] for further examples and for an analysis).

It is remarkable that ferromagnetic contributions to $\mathrm{J}_{12}\left(2 \mathrm{~K}_{12}\right.$, Eqn. (15b)) seem to be described realistically by the LFDFT procedure and our results show that these terms could be indeed rather important (as large as $0.061 \mathrm{eV}$ in the chosen example). Such terms have been neglected in earlier studies [29] or deemed to be small by physicists [24].

\subsection{Metal-Ligand and Metal-Metal Bonding in $\mathrm{Re}_{2} \mathrm{Cl}_{8}{ }^{2-}$}

The discovery of a strong $\mathrm{Re}-\mathrm{Re}$ bond in the $\mathrm{Re}_{2} \mathrm{Cl}_{8}{ }^{2-}$ anion in 1965 [30-32], termed quadruple bond, opened a new area in inorganic chemistry. Moreover, it contributed to initiate studies which helped to understand and to validate our knowledge about the chemical bond, based on the classical paper by Heitler-London [33] and on the Coulson-Fischer description of the twoelectron bond in the $\mathrm{H}_{2}$ molecule [34]. An excellent review of all developments covering both experiment and theory on the $\delta$ bond in the $\mathrm{Re}_{2}{ }^{6+}$ and $\mathrm{Mo}_{2}{ }^{4+}$ cores along with reference to original work has been published recently [35].

To analyze the $\mathrm{Re}-\mathrm{Re}$ bond in $\mathrm{Re}_{2} \mathrm{Cl}_{8}{ }^{2-}$ it is reasonable to start from the two square pyramidal $\mathrm{ReCl}_{4}{ }^{2-}$ fragments. For this coordination, the $15 \mathrm{~d}>$ orbitals of Re are split into $6 \mathrm{a}_{1}\left(\mathrm{~d}_{\mathrm{z} 2}\right), 2 \mathrm{~b}_{2}\left(\mathrm{~d}_{\mathrm{xy}}\right), 6 \mathrm{e}\left(\mathrm{d}_{\mathrm{xz}, \mathrm{yz}}\right)$, and $4 \mathrm{~b}_{1}$ $\left(d_{x 2-y 2}\right)$ species whose energies and compositions are depicted in Fig. 4 (left). The $\mathrm{d}_{\mathrm{z} 2}$ orbital is antibonding, but is largely stabilized by the $5 d-6$ s mixing which pushes it down, thus making it lowest in energy. This mixing is such that it increases the lobes along the axial direction and thus enhances the $\mathrm{Re}-\mathrm{Re}$ overlap in the dimer. It follows that the $\mathrm{Re}-\mathrm{Cl}$ bonding in the $\mathrm{ReCl}_{4}^{-}$fragment, which leads to $5 \mathrm{~d}-6 \mathrm{~s}$ hybridization has an indirect enforcing effect on the $\mathrm{Re}-$ $\operatorname{Re} \sigma$-bond. The energies of the $6 \mathrm{e}$ and $2 \mathrm{~b}_{2}$ orbitals of the $\mathrm{ReCl}_{4}^{-}$unit indicate a strong $\mathrm{Re}-\mathrm{Cl} \pi$-bonding interaction (out-of-plane and in-plane interactions with respect to the $\mathrm{ReCl}_{4}^{-}$plane for $6 \mathrm{e}$ and $2 \mathrm{~b}_{2}$, respectively), which are calculated to lie at almost the same energy. They give rise to $\pi$ and $\delta$ $\mathrm{Re}-\mathrm{Re}$ bonds, respectively. All four orbitals, $6 a_{1}, 2 b_{2}$, and $6 e$ are singly occupied in $\mathrm{ReCl}_{4}{ }^{2-}$ and yield four bonds between the two $\mathrm{ReCl}_{4}^{-}$units: one $\sigma$, two $\pi$ and one $\delta$ bond. A rough measure for the strength of these bonds are the spittings of the $a_{1}\left(9 a_{1}\right.$, $\left.12 \mathrm{a}_{1}\right), \mathrm{e}(11 \mathrm{e}, 12 \mathrm{e})$, and $\mathrm{b}_{2}\left(3 \mathrm{~b}_{2}, 4 \mathrm{~b}_{2}\right)$ orbitals, which are calculated to be $5.42,3.58,0.70$ (Fig. 4, right), thus reflecting a decrease of bond strength from $\sigma$ to $\pi$ to $\delta$.

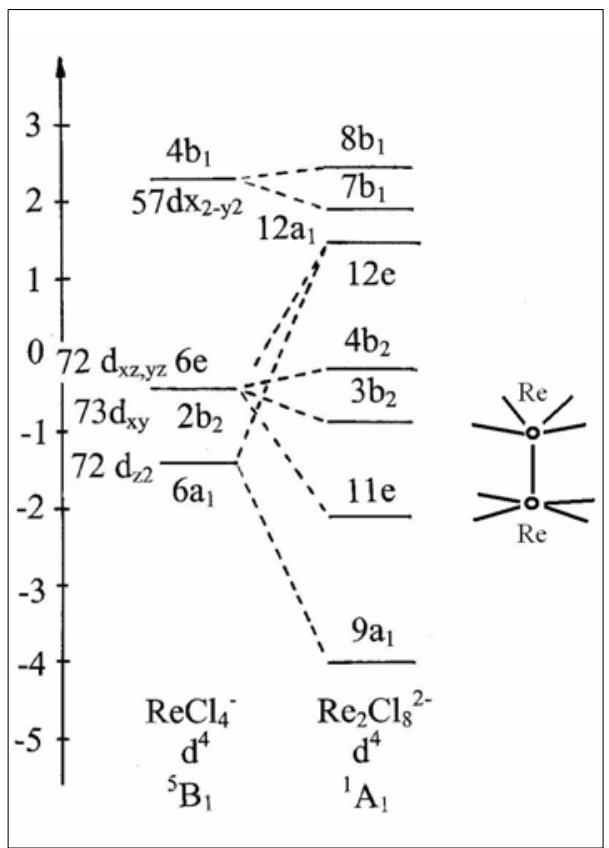

Fig. 4. Kohn-Sham MO-energy diagram for $\mathrm{Re}_{2} \mathrm{Cl}_{8}{ }^{2-}$ and its correlation with the KS-MO levels dominated by $5 \mathrm{~d}$ orbitals and their percentages of the constituting $\mathrm{ReCl}_{4}^{-}\left(\mathrm{C}_{4 v}\right.$ symmetry) fragment. For the sake of a better comparison, KS-MO energies for each cluster have been plotted taking their baricenter energy as a reference. Electronic ground state notations refer to $\mathrm{C}_{4 \mathrm{v}}$ (common symmetry for $\mathrm{ReCl}_{4}^{-}$and $\mathrm{Re}_{2} \mathrm{Cl}_{8}{ }^{2-}$ ).

This is clearly manifested by the plots of the electronic localization function (ELF) [36] (Fig. 5) which takes values between 1 (electron localization) and 0 (no localization) and thus reflects the concentration of charge into bonding or non-bonding domains. Thus, while the plot in Fig. 5a does not show any indication of accumulation of electron charge between the Re nuclei, the symmetry partitioned ELF plots (Fig. $5 b, c)$ nicely reflect this. The spectacular
Fig. 3. Correlation diagram between the energies of single determinants from DFT and the resulting multiplets of relevance for the magnetic exchange coupling in a $\left[\mathrm{Cu}\left(\mathrm{NH}_{3}\right)_{2}(\mathrm{OH})\right]_{2}{ }^{2+}$ model cluster with a ferromagnetic spin alignment. Model parameters for the calculation of the diagram (right), deduced from the DFT SD energies $E_{1}, E_{2}, E_{3}, E_{4}(-4.434,-3.798,-4.692$, $-4.238 \mathrm{eV}$, diagram left) are $\mathrm{K}_{12}=0.061, \mathrm{t}_{12}=0.159, \mathrm{U}=0.909$. 
feature of these plots is the $\sigma$-bond pathway which shows a bond-localization attractor between the Re nuclei but not only. Indeed, the plot for $\pi$ symmetry reflects a much weaker yet non-negligible bonding effect, while the one for $\delta$ does not display any bonding features. Apparently, the $\delta$-bonding in $\mathrm{Re}_{2} \mathrm{Cl}_{8}{ }^{2-}$ can be regarded as a weak bond which might as well be considered as a strong antiferromagnetic coupling (see below). This interaction can be fully destroyed when going from the eclipsed $\left(D_{4 h}\right)$ to the staggered $\left(D_{4 d}\right)$ conformation. For this latter geometry, the $\delta$-orbitals are rotated by $45^{\circ}$ with respect to each other, leading to strict orthogonality and to ferromagnetism. This could be achieved by chemical tuning [35]. The extended transition state (ETS, [37][38]) energy decomposition analysis lends support to this interpretation based on MO analysis and ELF plots (Table 1). In this analysis the interaction energy between two $\mathrm{ReCl}^{4-}$ fragments is partitioned into an electrostatic energy term $\Delta \mathrm{E}_{\text {Elstat }}$, the exchange (Pauli) repulsion energy $\Delta \mathrm{E}_{\text {Pauli }}$ and orbital interaction term $\Delta \mathrm{E}_{\text {orb }}$, which is further subdivided into terms pertaining to each orbital symmetry. The absence of $\delta$-bonds in the $\mathrm{D}_{4 \mathrm{~d}}$ geometry also explains the larger stability (by $-0.65 \mathrm{eV}$ for $\Delta \mathrm{E}_{\text {int }}$ ) of the eclipsed compared to the staggered form. The $\Delta \mathrm{E}_{\text {int }}$ energy change when going from the $\mathrm{D}_{4 \mathrm{~h}}$ to the $\mathrm{D}_{4 \mathrm{~d}}$ complex is a result of the balance between the $\Delta \mathrm{E}_{\text {Pauli }}$ term (which is in favor for the $\mathrm{D}_{4 \mathrm{~d}}$ geometry, $\left.\delta \Delta \mathrm{E}_{\text {Pauli }}=-0.59 \mathrm{eV}\right)$ and $\Delta \mathrm{E}_{\text {orb }}\left(\delta \Delta \mathrm{E}_{\text {orb }}=\right.$ $1.02)$, and to a lesser extend to the $\Delta \mathrm{E}_{\mathrm{Elstat}}$ term $\left(\delta \Delta \mathrm{E}_{\text {Elstat }}=0.23 \mathrm{eV}\right.$, i.e. both $\Delta \mathrm{E}_{\text {orb }}$ and $\Delta \mathrm{E}_{\text {Elstat }}$ are in favor of the $\mathrm{D}_{4 \mathrm{~h}}$ geometry). It is interesting to note that all contributions to $\Delta \mathrm{E}_{\text {orb }}$ become less negative when going from $\mathrm{D}_{4 \mathrm{~h}}$ to $\mathrm{D}_{4 \mathrm{~d}}$. However, reduction in bond strength in this direction is dominated by $\delta\left[\delta \Delta \mathrm{E}_{\text {orb }}(\delta)=0.72 \mathrm{eV}\right]$, followed by $\pi$ and then by $\sigma\left[\delta \Delta \mathrm{E}_{\text {orb }}(\pi)=\right.$ 0.19 and $\left.\delta \Delta \mathrm{E}_{\text {orb }}(\sigma)=0.11 \mathrm{eV}\right]$.

Yet another possibility to analyze Re$\operatorname{Re} \sigma, \pi$, and $\delta$ bonds within DFT is to apply our extended LFDFT model to each of them. This can easily be done for $\sigma$ and $\delta$ symmetry because for each type of bonding there are two MOs and two electrons available in $\mathrm{D}_{4 \mathrm{~h}}$ symmetry. This allows an analysis along the lines of a homonuclear diatomic m.o. problem for each of the two bonding modes separately. This is similar to a discussion of bonding in these systems in which $\delta$ electrons are being considered as decoupled from the $\pi$ and $\sigma$-electrons (Bursten and Clayton [39]). For $\pi$-bonds, there are four electrons and four orbitals which makes the analysis cumbersome. However, also in this case, an approximate treatment can be given, restricting the consideration to two electrons distributed over only two orbitals - bonding and antibond-
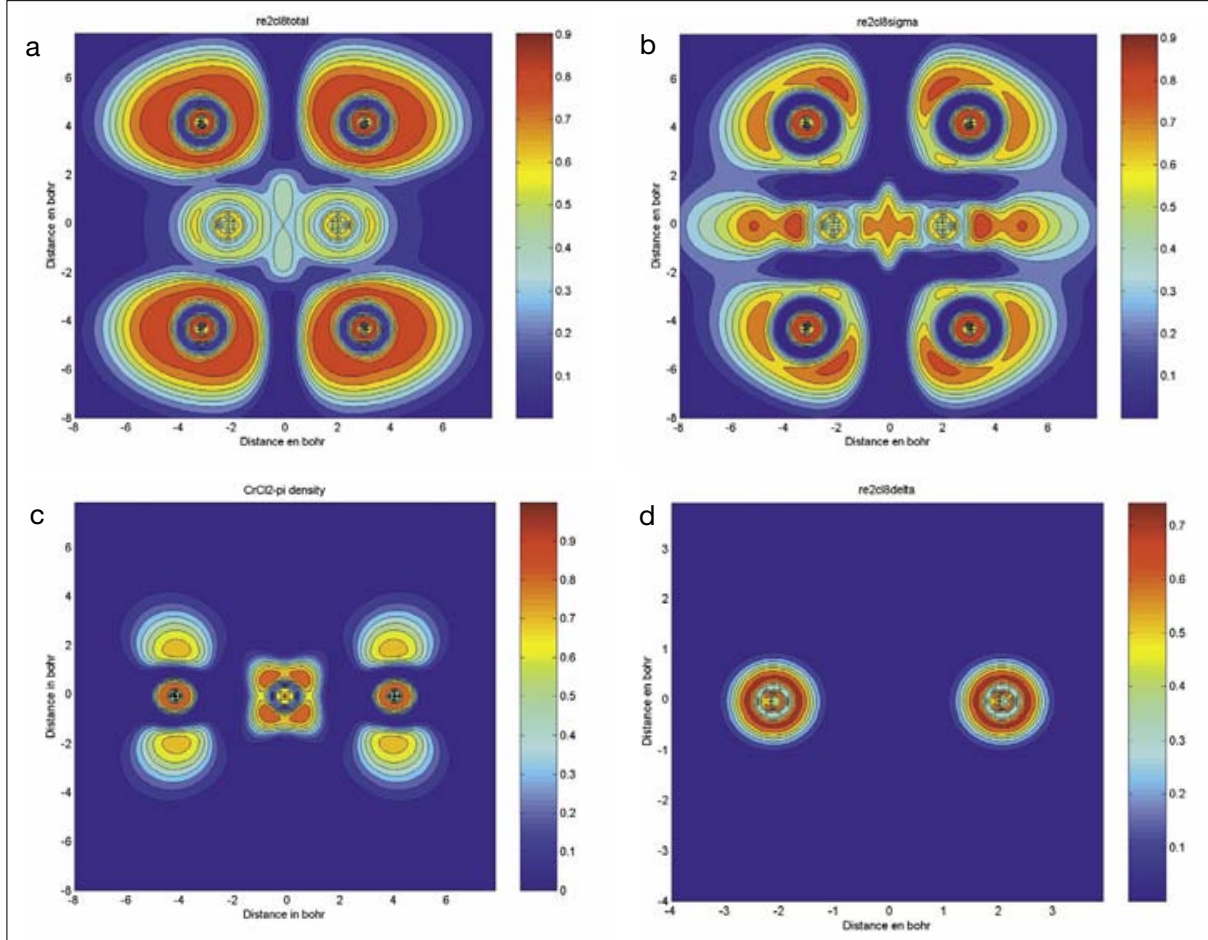

Fig. 5. Electronic localization function for $\mathrm{Re}_{2} \mathrm{Cl}_{8}{ }^{2-}$ taken within a plane containing the $\mathrm{Re}-\mathrm{Re}$ bond and four $\mathrm{Cl}$ ligands belonging to the constituting $\mathrm{ReCl}_{4}{ }^{-}$fragments. Contour diagrams have been plotted using the total density (a); the Re-Re $\sigma$-density $-a_{1}\left(C_{4 v}\right)$ symmetry (b); the Re-Re $\pi$-density $-e\left(C_{4 v}\right)$ symmetry (c); and Re-Re $\delta$-density $-b_{2}\left(C_{4 v}\right)$ symmetry (d). See Fig. 4 for symmetry notations and a correlation diagram within the $\mathrm{C}_{4 \mathrm{v}}$ subgroup, common for the dimer $\mathrm{D}_{4 \mathrm{~h}} \mathrm{Re}_{2} \mathrm{Cl}_{8}{ }^{2-}$ and the $\mathrm{ReCl}_{4}{ }^{-}$fragment.

Table 1. The unique bonding situation in $\mathrm{Re}_{2} \mathrm{Cl}_{8}{ }^{2-}$ with a bonding energy partitioned with respect to two non-interacting $\mathrm{Re}_{2} \mathrm{Cl}_{4}{ }^{1-}$ sub-units in its eclipsed (ideal for $\delta$-bonding) and its staggered ( $\delta$-bonding is abolished) conformations ${ }^{a}$

$\begin{array}{ccccccccc}\Delta \mathrm{E}_{\text {Pauli }} & \Delta \mathrm{E}_{\text {Elstat }} & \Delta \mathrm{E}_{\text {orb }} & \Delta \mathrm{E}_{\text {int }} & \Delta \mathrm{E}_{\text {orb }}\left(\mathrm{a}_{1}\right) & \Delta \mathrm{E}_{\text {orb }}\left(\mathrm{a}_{2}\right) & \Delta \mathrm{E}_{\text {orb }}\left(\mathrm{b}_{1}\right) & \Delta \mathrm{E}_{\text {orb }}\left(\mathrm{b}_{2}\right) & \Delta \mathrm{E}_{\text {orb }}(\mathrm{e}) \\ \begin{array}{c}\text { eclipsed } \\ \left(\mathrm{D}_{4 \mathrm{~h}}\right)\end{array} & & & & & & & \\ 25.46 & -10.31 & -20.79 & -5.64 & -10.63 & 0.00 & -0.07 & -0.80 & -9.29\end{array}$

staggeredc

$\left(D_{4 d}\right)$

24.87

$\begin{array}{llll}-10.08 & -19.77 & -4.99 & -10.52\end{array}$

0.00

$-0.08$

$-0.08$

$-9.10$

a Scalar relativistic ZORA calculations

b $9 a_{1}{ }^{2}(\sigma) .11 \mathrm{e}(\pi)^{4} \cdot\left(3 \mathrm{~b}_{2}, 4 \mathrm{~b}_{2}(\delta)\right)^{2}$ - singlet ground state

c $9 \mathrm{a}_{1}{ }^{2}(\sigma) \cdot 11 \mathrm{e}(\pi)^{4} \cdot\left(3 \mathrm{~b}_{2}, 4 \mathrm{~b}_{2}(\delta)\right)^{2}$ - triplet ground state; $\mathrm{C}_{4 \mathrm{v}}$ symmetry notations

ing, each of them transforming as one of the components of the doubly degenerate orbital e.

In Table 2 we include singlet triplet separations $\mathrm{J}_{12}$, corresponding to $\sigma, \pi$, and $\delta$ bonding, obtained from DFT calculations. $\mathrm{Re}-\mathrm{Re}$ bond energies decrease from $\sigma$ to $\pi$ to $\delta$ following the lines of the MO and the ETS analysis. It is interesting to note that the $\delta \varepsilon(\lambda)(\lambda=\sigma, \pi, \delta)$ splittings of the KS-MO energies and $J_{12}(\lambda)$ are very close in magnitude and nearly equal to the values of the hopping integral $t_{12}$. This reflects the common covalent origin of these parameters. At the same time, $\Delta \mathrm{E}_{\text {orb }}(\lambda)$ deduced from the ETS analysis are larger than $\mathrm{J}_{12}(\lambda)$ and $\delta \varepsilon(\lambda)$ (Table 2). Possibly polarization effects contribute to this difference. The parameter $\mathrm{K}_{12}$ is just the ferromagnetic exchange integral which may become operational in the limit of zero overlap. It leads to a triplet $\left({ }^{3} \mathrm{~A}_{2}\right)$ ground state in the staggered $\left(D_{4 d}\right)$ conformation of $\mathrm{Re}_{2} \mathrm{Cl}_{8}{ }^{2-}$ where $\delta$ bonding is fully suppressed.

\section{Conclusions}

The LFDFT models developed in our group turn out to be able to calculate electronic multiplet structures as well as fine 
Table 2. Singlet versus triplet ground state stabilizations in eclipsed $\mathrm{Re}_{2} \mathrm{Cl}_{8}{ }^{2-}$ based on a two-electrontwo-center bond model within the single determinant DFT approach of DFT for $\sigma, \pi$ and $\delta$ bonds $^{a}$

$\begin{array}{cccc}\lambda= & \sigma & \pi & \delta \\ \mathrm{J}_{12}=\mathrm{E}_{-}-\mathrm{E}_{\mathrm{T}} & -5.259 & -3.470 & -0.461 \\ \delta \varepsilon(\lambda) & (-4.769) & (-3.125) & (-0.419) \\ \Delta \mathrm{E}_{\text {orb }}(\lambda) & -5.41 & -3.58 & -0.704 \\ & -10.633 & -9.287 & -0.802 \\ \mathrm{~K}_{12} & 0.131 & 0.060 & 0.004_{5} \\ & (0.100) & (0.068) & \left(0.004_{5}\right) \\ \mathrm{t}_{12} & 5.714 & 3.757 & 0.719 \\ & (5.192) & (3.406) & (0.656) \\ \mathrm{U} & 0.391 & 0.340 & 0.630 \\ & (0.456) & (0.296) & (0.576)\end{array}$

a Scalar relativistic (non-relativistic, in parenthesis) spin-unrestricted ZORA calculations in $D_{4 h}$ symmetry with the following configurations for the Re d-orbitals for $\sigma, \pi$ and $\delta$ bonding: $\left(a^{+} a^{-}\right)=$ $6 \underline{a}_{1 q}{ }^{2} 6 \underline{e}_{1 u}{ }^{4} 2 \underline{b}_{2 g}{ }^{2} ;\left(b^{+} b^{-}\right)=6 \underline{a}_{2 u}{ }^{2} 6 e_{1 u}{ }^{4} 2 b_{2 g}{ }^{2}, 6 a_{1 q}{ }^{2} 6 e_{1 u}{ }^{2} 6 \underline{e}_{q}{ }^{2} b_{2 g}{ }^{2}, 6 a_{1 q}{ }^{2} 6 e_{1 u}{ }^{4} 2 \underline{b}_{1 u}{ }^{2} ;\left(a^{+} b^{+}\right.$and $\left.a^{+} b^{-}\right)=$ $6 \underline{a}_{1 g}{ }^{1} 6 a_{2 u}{ }^{1} 6 e_{1 u}{ }^{4} 2 b_{2 g}{ }^{2} ; 6 a_{1 g}{ }^{2} 6 \underline{e}_{1 u}{ }^{3} 6 e_{g}{ }^{1} 2 b_{2 g}{ }^{2} ; 6 a_{1 g}{ }^{2} 6 e_{1 u}{ }^{2} 2 \underline{b}_{2 g}{ }^{1} 2 \underline{b}_{1 u}{ }^{\top}$ (a,b orbitals under consideration are underlined). Values of the transfer(hopping) integral $t_{12}$, the Heisenberg exchange integral $\left(K_{12}\right)$ and the effective transfer energy $(U)$ are also included. $\operatorname{Re}-\operatorname{Re}(2.236 \AA)$ and $\operatorname{Re}-\mathrm{Cl}(2.331 \AA)$ bond distances are obtained from LDA-DFT geometry optimizations.

structures not only for single nuclear, but also in dinuclear TMI complexes. In this case, not only magnetic exchange, but also the metal-metal chemical bond can be interpreted and well understood in terms of interactions between orbitals in the active space of the d-electrons. They can be used to interpret bonding and magnetic phenomena on the same footing and are valuable in addition to other methods for the study of the chemical bond, e.g. the extended transition state method, the electron localization function and analysis of the Laplacian of the electron density (Bader analysis). Following a more general, symmetry-based formalism [40] the method can be extended to charge transfer spectra. Its ability to also treat spectra of rare-earth and actinide complexes is presently being explored in our group [41].

\section{Acknowledgement}

This study was supported by the Swiss National Science Foundation, the Bundesamt für Forschung and Wissenschaft (Swisse) and a COST Action of the European Science Foundation.

Received: May 19, 2005

[1] M. Atanasov, C.A. Daul, C. Rauzy, Chem. Phys. Lett. 2003, 367, 737.

[2] M. Atanasov, C.A. Daul, C. Rauzy, Structure and Bonding 2004, 106, 97.

[3] C.A. Daul, Chimia 2004, 58, 316.

[4] P. Hohenberg, W. Kohn, Phys. Rev. 1964, 136, B864.

[5] W. Kohn, L.J. Sham, Phys. Rev. 1965, 140, A1133.

[6] T. Ziegler, Chem. Rev. 1991, 91, 651.

[7] W. Koch, M.C. Holthausen, in 'AChemist's Guide to Density Functional Theory', Wiley-VCH, Weinheim, 2000.
[8] C.C.J. Roothaan, Rev. Mod. Phys. 1960, 32, 179.

[9] a) G. te Velde, F.M. Bickelhaupt, E.J. Baerends, C. Fonesca Guerra, S.J.A. van Gisbergen, J.G. Snijders, T. Ziegler, J. Comput. Chem. 2001, 22, 931; b) ADF program, release ADF2004.01, web site: http://www.scm.com.

[10] a) G. Vignale, M. Rasolt, Phys. Rev. Lett. 1987, 59, 2360; b) G. Vignale, M. Rasolt, Phys. Rev. B 1988, 37, 10685.

[11] F.D. Sala, A. Görling, J. Chem. Phys. 2003, 118, 10439.

[12] R.J. Deeth, in 'Comprehensive Coordination Chemistry II', Eds. J.A. McCleverty, T.J. Meyer, Vol. Ed. A.B.P. Lever, Elsevier, 2003, Vol. 2, p. 439.

[13] T. Schönherr, M. Atanasov, H. Adamsky, in 'Comprehensive Coordination Chemistry II', Eds. J.A. McCleverty, T.J. Meyer, Vol. Ed. A.B.P. Lever, Elsevier, 2003, Vol. 2, p. 443.

[14] M.F. Hazenkamp, H.U. Güdel, M. Atanasov, U. Kesper, D. Reinen, Phys. Rev. B 1996, 90, 2367.

[15] U. Oetliker, M. Herren, H.U. Güdel, U. Kesper, C. Albrecht, D. Reinen, J. Chem. Phys. 1994, 100, 8656.

[16] T.C. Brunold, A. Hauser, H.U. Güdel, $J$. Lumin. 1994, 59, 321.

[17] M. Atanasov, J.-L. Barras, L. Benco, C.A. Daul, J. Am. Chem. Soc. 2000, 122, 4718.

[18] M. Atanasov, C. Rauzy, P. Baettig, C.A. Daul, Int. J. Quantum Chem., in press, published online on 06.12.2004.

[19] M. Atanasov, E.J. Baerends, P. Baettig, R. Bruyndonckx, C.A. Daul, C. Rauzy, M. Zbiri, Chem. Phys. Lett. 2004, 399, 433.

[20] M. Atanasov, C.A. Daul, C. Rauzy, P. Baettig, in preparation.

[21] M. Atanasov, C.A. Daul, E. Penka Fowe, in preparation.
[22] M. Atanasov, C.A. Daul, Chem. Phys. Lett. 2003, 379, 209.

[23] M. Atanasov, C.A. Daul, Chem. Phys. Lett. 2003, 381, 584.

[24] P.W. Anderson, Phys. Rev. 1959, 115, 2.

[25] P.J. Hay, J.C. Thibeault, R. Hoffmann, J. Am. Chem. Soc. 1975, 97, 4884.

[26] M. Atanasov, S. Angelov, Chem. Phys. 1991, 150, 383.

[27] D.J. Hodgson, Progr. Inorg. Chem. 1975, 19, 173.

[28] L. Noodleman, J. Chem. Phys. 1981, 74, 5737.

[29] R. Schenker, H. Weihe, H.U. Güdel, Inorg Chem. 1999, 38, 5593.

[30] F.A. Cotton, N.F. Curtis, B.F.G. Johnson, W.R. Robinson, Inorg. Chem. 1965, 4, 326.

[31] F.A. Cotton, C.B. Harris, Inorg. Chem. 1965, 4,330 .

[32] F.A. Cotton, Inorg. Chem. 1965, 4, 334.

[33] W. Heitler, F. London, Z. für Physik 1927, $44,455$.

[34] C.A. Coulson, I. Fischer, Philosophical Magazine 1949, 40, 386.

[35] F.A. Cotton, D.G. Nocera, Acc. Chem. Res. 2000, 33, 483.

[36] A.D. Becke, K.E. Edgecombe, J. Chem. Phys. 1990, 92, 5397.

[37] T. Ziegler, A. Rauk, Theor. Chim. Acta 1977, 46, 1 .

[38] F.M. Bickelhaupt, E.J. Baerends, in 'Reviews in Computational Chemistry', Vol. 15, Eds. K.B. Lipkowitz, D.B. Boyd, Wiley-VCH, John Wiley and Sons, Inc., New York, Chap. 1, 2000.

[39] B.E. Bursten, T.W. Clayton, Jr., J. Cluster Science 1994, 5, 157.

[40] C.A. Daul, Int. J. Quant. Chem. 1994, 52, 867.

[41] M. Atanasov, C.A. Daul, H.U. Güdel, T.A. Wesolowski, M. Zbiri, Inorg. Chem. 2005, in press. 